\title{
ARTIGOS
}

\section{SITUAÇÃO ATUAL DA DOENÇA DE CHAGAS NA GUIANA FRANCESA}

\section{Philippe Esterre e Jean-Pierre Dedet}

\begin{abstract}
Localizada entre dois focos endêmicos da doença de Chagas, a Venezuela e o Brasil, a Guiana Francesa conheceu poucos casos dessa afecção de 1940 até 1956.

Um inquérito sorologico, utilizando-se uma pesquisa ativa sobre pacientes cardiopáticos e uma pesquisa passiva, permitiu evidenciar a existência de dois casos autóctones. Apesar da presença de vetores domésticos, o risco da doença de Chagas na Guiana Francesa parece limitado à aparição episódica de escassos casos humanos.
\end{abstract}

\section{Palavras-chaves: Doença de Chagas. Guiana Francesa. Sorologia.}

Considera-se geralmente que a infecção pelo Trypanosoma cruzi atinge entre $10^{1}$ e mais de 20 milhões ${ }^{14}$ de pesssoas na América do Sul. A existência de focos endêmicos, bem delimitados geograficamente, já é conhecida há muito tempo ${ }^{16}$.

$\mathrm{Na}$ região amazônica, o nosso conhecimento da epidemiologia da doença de Chagas fica heterogêneo. Além do grande foco da Venezuela bem conhecido pela sua endemicidade elevada, não temos idéia da extensão da doença na Guiana e no Suriname. No Estado do Pará, o primeiro caso assinalado data de 1968 e desde essa época, sete novos casos autóctones foram encontrados novamente na região de Belém 1314.

$\mathrm{Na}$ Guiana Francesa, oito casos humanos foram relatados entre 1940 e 1956 , sendo o parasita identificado somente a partir de critérios morfológi$\cos ^{5} 6$. Sobre 160 soros examinados, obtidos de indios Oyampi originários da bacia do rio Oiapoque, três casos positivos foram identificados segundo um inquérito sorológico de 1971 utilizando imunofluorescência. Desde 1956 e até uma data muito recente ${ }^{2}$, não se havia registrado nenhum caso na Guiana Francesa.

$O$ presente trabalho faz um balanço na situação atual da doença na Guiana, dando detalhes a respeito da prevalência da infecção humana comprovada através de testes imunológicos e revelando a importância dos diversos ciclos ecológicos implicados.

\section{MATERIAL E MÉTODOS}

Uma sensibilização do corpo médico da Guiana, realizada ao inicio do ano de 1983 , permitiu-nos um inquérito soro-epidemiológico a partir de várias populações (Fig. 1).

Trabalho realizado no Instituto Pasteur da Guiana Francesa, Cayenne, Guiana Francesa.

Endereço para correspondência: Dr. Philippe Esterre, Institut Pasteur de Guyane Française, 97306 Cayenne Cedex, Guyane Française.

Recebido para publicação em 23/06/1986.

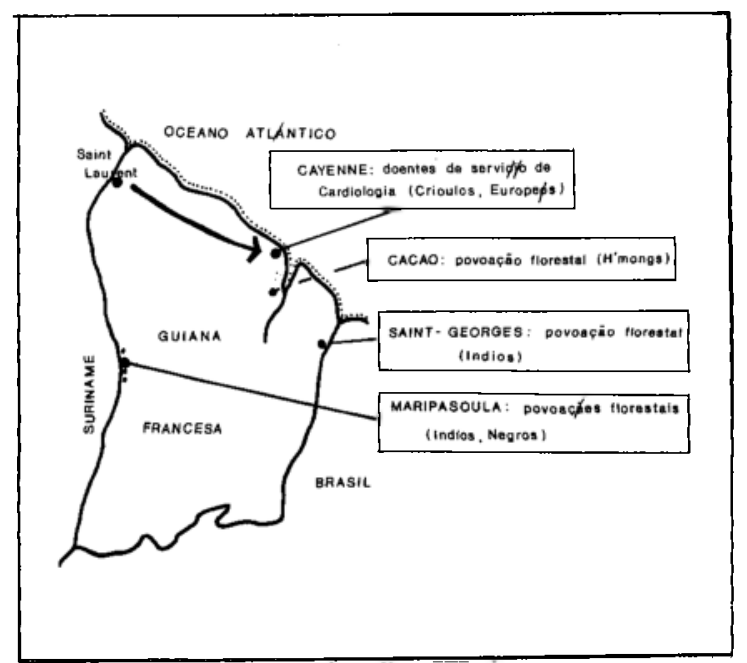

Fig. 1 -Populações estudadas no inquérito sorológico de doença de Chagas na Guiana Francesa.

Numa primeira população estudada foi feita uma pesquisa ativa: a amostra era constituida de pacientes atendidos no Serviço de Cardiologia do Hospital de Caiena, correspondendo ao critério de escolha a presença de insuficiência cardiaca crônica com perturbações bilaterais da condução. Trata-se de uma amostra envolvendo individuos doentes, com alta probabilidade de infecção chagásica ${ }^{2}$.

A segunda população estudada foi constituida de várias amostras de habitantes de povoações da mata, sobre as quais foi feita uma pesquisa passiva. Um primeiro grupo foi quase exclusivamente estabelecido a partir de refugiados H'mong recentemente chegados na Guiana, na povoação de Cacao (1977). Esta amostra envolve individuos sãos, sem imunidade contra as infecções pelos tripanosomatídeos (pelo menos na chegada à Guiana), mas com riscos de exposição, devidos à presença nos arredores da povoação de marsupiais comensais com alto nível de infecção. 
Esterre P. Dedet JP. Situação atual da doença de Chagas na Guiana Francesa. Revista da Sociedade Brasileira de Medicina Tropical 20: 139-142, Jul-Set, 1987.

A segunda pesquisa passiva teve por objetivoíndios das tribos Wayana e Emerollon vivendo em Aloïke, Twanké e Elahé, três povoações da mata perto de Maripasoula, negros da tribo Boni de Maripasoula e indios Palikur de Saint Georges de l'Oyapoque. Estes individuos estão sensibilizados desde a infância às leishmanioses e provavelmente expostos à infecção pelo Trypanosoma cruzi.

Alguns soros procedentes do banco de sangue de Caiena (crioulos e europeus) serviram de controle, representando o meio urbano.

Um lote de soros de doentes brasileiros, obtido no Instituto de Medicina Tropical de São Paulo, serviu de controle positivo.

Um pequeno inquérito sorológico também foi realizado sobre a população canina: a colheita foi feita sobre 14 animais entre os 17 cães recenseados na povoação.

\section{TÉCNICAS DE LABORATÓRIO}

Foi utilizada a reação de imunofluorescência indireta (IFI), sendo o antigeno constituido por um "pool" de três cepas de T.cruzi zimodema 1, isoladas na Guiana. A reaçāo foi efetuada concomitantemente com conjugados fluorescentes (Institut Pasteur Production) anti-imunoglobulinas (IgG) (cadeia y) e anti-IgM (cadeia $\mu$ ) humanas. No caso de um resultado ser positivo, uma amostra de soro era enviada a um laboratório de referência para verificação, em particular de uma fração antigênica 5 específica ${ }^{12}$.

No que diz respeito à análise dos soros de origem canina, a mesma reação de IFI foi realizada, utilizando conjugados fluorescentes anti-IgG de cão (Laboratórios Biosys).

\section{RESULTADOS}

\section{Distribuição e prevalência (Tabela 1)}

Entre 64 amostras de sangue de pacientes apresentando uma cardiopatia não obstrutiva sugerin- do um diagnóstico de doença de Chagas, um só doente, de origem guianesa, apresentou uma sorologia claramente positiva a $1 / 160$ (anticorpos de tipo IgG e IgM) comprovada por dois laboratórios de referência (IFI positiva a $1 / 160$ e teste Elisa positivo ${ }^{2}$; presença do arco 5\% específico).

Segundo o inquérito sorológico realizado a partir de 86 soros recolhidos na população de Cacao, só foram evidenciados 6 casos ao limiar da positividade (titulo de anticorpos IgG acima de 1/20). Este resultado deve ser correlacionado às possibilidades de reações cruzadas com uma leishmaniose. Esta hipótese foi comprovada por um exame laboratorial (IFI com um composto de anti-Ig totais e um "pool" de cepas de Leishmania braziliensis guyanensis servindo de antígeno) e, em certos casos, um quadro clínico típico de leishmaniose cutânea ("Pian-bois").

No caso dos indivíduos das povoações da mata, esse tipo de reação cruzada fica mais freqüente, já que sobre 180 soros examinados, foram classificados 22 como duvidosos, e somente um francamente positivo. Este último resultado foi comprovado por um laboratório de referência (titulo dos anticorpos anti-IgG positivo a $1 / 160$ e presença de um arco 5 ? específico). A totalidade dos casos duvidosos apresentou um sorodiagnóstico de leishmaniose positivo.

\section{$A$ doença humana}

Foram relatados entre 1940 e 1956 oito casos da doença de Chagas nas publicações do Instituto Pasteur da Guiana Francesa ${ }^{5} 67$. Sete indivíduos apresentaram forma aguda e um apresentou cardiopatia chagásica crônica. Nenhum caso foi diagnosticado entre 1956 e 1984.

O primeiro doente do nosso inquérito apresentando uma reação nitidamente positiva era um agricultor crioulo de condição modesta, morando numa cabana feita de palmas de coqueiro. Faleceu recentemente de uma septicemia devida a uma shigelose intestinal grave. A autópsia revelou lesões cardiacas patognomônicas de uma insuficiência cardiaca ligada a doença de Carlos Chagas: cardiomegalia

Tabela 1 -Inquérito sorológico de doença de Chagas na Guiana Francesa no periodo 1983-1985.

\begin{tabular}{lcccc}
\hline & $\begin{array}{c}\text { Total das } \\
\text { amostras }\end{array}$ & Negativas & Duvidosas & Positivas \\
\hline $\begin{array}{l}\text { Cayenne - Saint Laurent } \\
\text { (pesquisa ativa) }\end{array}$ & 64 & 57 & 6 & 1 \\
\hline Cayenne & 35 & 35 & 0 & 0 \\
Cacao & 86 & 80 & 6 & 0 \\
Saint Georges & 7 & 6 & 1 & 0 \\
Maripasoula & 173 & 150 & 22 & 1 \\
\hline Total de pesquisa passiva & 301 & 271 & 29 & 1 \\
\hline
\end{tabular}


global e estreitamento considerável da parede do ventrículo esquerdo, e presença de um importante aneurisma apical. Trata-se efetivamente de um caso autóctone de doença de Chagas, o primeiro evidenciado por métodos modernos de imunologia na Guiana Francesa. Foi apresentada essa observação numa prévia publicação².

O segundo indivíduo, apresentando uma nítida positividade, era uma jovem Boni de 18 anos, vivendo na mata afastada. Grávida, e aparentemente em boa saúde no dia da tomada de sangue, essa pessoa foi em seguida perdida de vista pelo médico do setor, o que nos impediu confirmar a existência da infeç̧ão chagásica.

\section{Existência de um ciclo peridoméstico}

A presença de $T$. cruzi foi assinalada na Guiana Francesa em três espécies de marsupiais: Didelphis marsupialis ${ }^{4}$, Philander (= Metachirops) opossum e Caluromys philander 4 . Um recente inquérito evidenciou uma taxa elevada de infecção dos Didelphis pelo T.cruzi (acerca de $31 \%$ de animais positivos). $\mathrm{Na}$ povoação florestal de Cacao particularmente, onde se estabeleceu, alguns anos atrás, uma importante colônia de refugiados do Laos, a prevalência da infecção em Didelphis atinge $42,8 \%{ }^{2}$. Este resultado nos incitou a efetuar um inquérito sorológico na população canina da povoação, no intento de confirmar a existência de um ciclo peridoméstico ativo. Dos 14 cães amostrados, três apresentaram taxas sorológicas em termos de IFI compativeis com uma infecção pelo $T$. cruzi (positividade acima de 1/40) tanto mais que eram baixas, ou nulas, as taxas sorológicas anti-Leishmania. Sendo a origem dos cães de Cacao estritamente local e a leishmaniose visceral ausente na Guiana, pode-se confirmar a infecção latente de certos animais domésticos pelo $T$. cruzi.

\section{COMENTÁRIOS}

O programa de pesquisa sobre os reservatórios e vetores da doença de Chagas na Guiana Francesa foi iniciado no ano de 1982, quando se descobriu $T$. cruz $i$ por ocasião de estudos a respeito da infeç̧ão natural de mamíferos selváticos pela Leishmania.

Os primeiros trabalhos evidenciaram a prevalência elevada das infecções pelo T.cruzi nos marsupiais silvestres da Guiana, particularmente em D. marsupialis. Foi também evidenciada a existência de seis espécies de triatomineos vetores (alguns formando pequenas colônias no interior mesmo de certas habitações na cidade de Caiena ${ }^{3}{ }^{4}$ ). Conseqüentemente, interrogamonos quanto às razões da aparente ausência de casos humanos durante os últimos trinta anos.

É recomendado o uso de métodos imunológicos modernos nos inquéritos de campo da doença de Cha- gas, sendo os testes IFI e Elisa os mais sensiveis ${ }^{8} 1014$. $\hat{E}$ bastante representativa da situação real a pesquisa imunológica, permanecendo sorologicamente positivo por toda a vida, um doente não tratado. A descoberta recente na ocasião da nossa pesquisa de um caso autóctone de doença de Chagas, o primeiro desde 1956, comprova que na Guiana Francesa, essa afeç̧ão não pertence só ao passado. A fraca incidência evidenciada nas populaçōes estudadas ( 3,3 por 1000 ) fica muito próxima das taxas encontradas na população de Belém (2,7 por 1000) ${ }^{9}$. Esses dados parecem indicar que nessas regiōes o risco é limitado à aparição episódica de escassos casos humanos. Podese enumerar um certo número de fatores para explicar a ausência de antropização dessa zoonose na Guiana Francesa: baixa densidade populacional no território, nivel de vida bastante alto, utilização de material artificial na construção. Tomando em conta a hipótese de Lainson e cols ${ }^{9}$, segundo a qual é a ausência de espécies domésticas que limita a extensão da doença de Chagas no Pará, a situação contrária na Guiana Francesa impõe uma estrita vigilância sanitária $e$ epidemiológica da afecção. A existência de um ciclo peridoméstico numa povoação florestal incita-nos a aumentar essa vigilância.

\section{SUMMARY}

Although located between two endemic areas of Chagas' disease (Venezuela and Brazil), French Guiana recorded only few cases of this infection between 1940 and 1956. A serological survey, using either active detection in cardiopathic patients or a passive one resulted in the finding of two autochthonous cases. In spite of the presence of a peridomiciliary cycle the risk of Chagas' disease in French Guiana seems to be limited to the episodic occurrence of rare sporadic human cases. Serology.

Key words: Chagas' disease. French Guiana.

\section{AGRADECIMENTOS}

Agradecemos ao Prof. D. Afchain (Instituto Pasteur de Lille, França, Prof. A. Capron) pela realização do teste de competição em Elisa a partir de um anticorpo monoclonal antiantigeno 5 , especifico de T. cruzi; ao Sr. M. Tibayrenc (IBBA, La Paz, Bolivia) pela realização da caracterização enzimática das cepas; ao Prof. M.E. Camargo (Instituto de Medicina Tropical de São Paulo, Brasil) que nos forneceu as amostras de soros positivos; à Sra. F. Gay que assegurou o cultivo das cepas no laboratório; ao Dr. M. J. Maitre (veterinário) que nos permitiu efetuar a colheita nos cães de Cacao, também como ao Sr. e Sra. F. e M. Guerrin pela tradução do manuscrito. 


\section{REFERÊNCIAS BIBLIOGRÁFICAS}

1. Barretto MP. Epidemiologia. In: Trypanosoma cruzi e doença de Chagas. Brener $\mathbf{Z}$ Andrade $\mathbf{Z}$ (Eds), Rio, Brasil, 1979.

2. Beaudet B, Esterre P, Jacquet-Vialet P, Ganty J, Dedet JP. Un cas de cardiopathie chagasique chronique autochtone en Guyane française. Bulletin de la Société de Pathologie Exotique 78:56-63, 1985.

3. Chippaux JP, Pajot FX, Geoffroy B, Tavaklian G. Etude préliminaire sur l'écologie et la systématique des triatomes (Hemiptera, Reduviidae) de Guyane française. Cahiers Orstom, série Entomologie Médicale et Parasitologie 23: 75-85, 1985.

4. Dedet JP, Chippaux JP, Goyot P, Pajot FX, Tibayrenc M, Geoffroy B, Gosselin H, Jacques-Vialet P. Les hôtes naturels de Trypanosoma cruzi en Guyane française. Endémicité élevée du zymodème 1 chez les marsupiaux sauvages. Annales de Parasitologie Humaine et Comparée 60: 111-117, 1985.

5. Floch H, Boulan J. Sur deux nouveaux cas de schizotrypanosomiase humaine américaine en Guyane française. Archives de l'Institut Pasteur de Guyane Française et du Territoire de l'Inini 412: 1-5, 1956.

6. Floch H, Camain R. Deux nouveaux cas de maladie de Chagas en Guyane française. Bulletin de la Société de Pathologie Exotique 41: 347-350, 1948.

7. Floch H, Lajudie P. Recherches sur la trypanosomiase humaine américaine en Guyane française. Rhodnius prolixus et $R$ hodius pictipes, vecteurs naturels de choix de $T$. cruzi. Archives de l'Institut Pasteur de Guyane Française et du Territoire de l'Inini 117: 1-5, 1945.
8. Fuchs AP, Fioratti VL, Mello VA, Boainain E. Diagnóstico sorológico na doença de Chagas, estudo comparativo de diferentes técnicas. Revista do Instituto de Medicina Tropical de São Paulo 22: 242-245, 1980.

9. Lainson R, Shaw JJ, Fraiha H, Miles MA, Draper CC. Chagas'disease in the Amazon basin: T. cruzi infections in sylvatic mammals, triatomine bug and man in the state of Pará, North Brasil. Transactions of the Royal Society of Tropical Medicine and Hygiene 73: 193-204, 1979.

10. Lainson R, Shaw JJ, Naiff RD. Chagas'disease in the Amazon basin: speculations on transmissions per os. Revista do Instituto de Medicina Tropical de São Paulo 22: 294-297, 1980.

11. Larrouy G, Brochier B, Dos Santos LG, Queiroz SF, Magnaval JF. Les méthodes ELISA pour le dépistage des sujets porteurs de $T$. cruzi, étude comparative. Bulletin de la Société de Pathologie Exotique 76: 553558, 1983.

12. Lemesre JL, Breniere F. Characterization of a specific 5 antigen of $T$. cruz $i$ in culture medium. Diagnosis application. In: Abstracts of the 5 th International Congress of $\mathrm{Pa}$ rasitology, 1982.

13. OMS. WHO scientific group on cell-mediated immunity and resistance to infection. WHO reports séries 519 , 1974.

14. OMS. Workshop on epidemiological, social and economic aspects of present and future methods of Chagas' disease control. WHO report 1980.

15. Série C. Activités épidémiologiques. Archives de l'Institut Pasteur de Guyane Française 530: 45-73, 1971.

16. Wilcocks C, Manson-Bahr PEC. Manson's Tropical Diseases. Ed Baillière Tindell, London, 1978. 